\title{
Joint Council on Thoracic Surgical Education: An investment in our future
}

\author{
Edward D. Verrier, MD
}

The Joint Council on Thoracic Surgery Education, Inc, was established in 2008 with the mission "to advance thoracic surgery education." The American Association for Thoracic Surgery (AATS), American Board of Thoracic Surgery (ABTS), Society of Thoracic Surgery (STS), and Thoracic Surgery Foundation for Research and Education were the 4 founding organizations that equally contributed to the creation of the Joint Council. The focus to date has been on resident and postgraduate thoracic surgery education.

Current surgical residency models in the United States can be traced back to their origins at Johns Hopkins University at the turn of the 20th century. William Halsted and William Osler each contributed to the concept that surgical training was essentially an advanced apprenticeship model based on scientific foundation, defined structure, standardization of training, bedside and operating room teaching, graded responsibility, and residents at different levels working together. ${ }^{1}$ Surgical training was initially pyramidal in design with multiple entering interns being exposed to a broad-based curriculum culminating in a few specialized surgical "chief" residents. The "master-apprenticeship" model persisted until the 1930s with the first formal residency in thoracic surgery being established at the University of Michigan in 1928 by John Alexander. This Hopkins resident education model was successful, but Edward Churchill at the Massachusetts General Hospital took the next steps forward with the recommendations to create a more rectangular organization structure for resident training. ${ }^{2}$ Churchill emphasized most of the principles of surgical education that persist today: avoidance of exploitation of the intern, exposure to basic science, staff appointment to qualified surgical educators, flexibility in training when possible, graded responsibility, and the creation of an overall advisory board for graduate education. This led to the creation of the first Committee on Graduate Surgery Training in 1934 and the subsequent implementation of the American Board of Surgery in 1937, the Board of Thoracic Surgery in 1948, and the independent ABTS in 1971. This

\footnotetext{
From the Department of Surgery, University of Washington, and Surgical Director, Joint Council of Thoracic Surgery Education, Inc, Seattle, Wash.

Disclosures: Author has nothing to disclose with regard to commercial support.

Received for publication Aug 16, 2010; revisions received Oct 6, 2010; accepted for publication Oct 14, 2010.

Address for reprints: Edward D. Verrier, MD, University of Washington, Surgery, 1959 NE Pacific St, Box 356310, Seattle, WA 98195 (E-mail: edver@u. washington.edu).

J Thorac Cardiovasc Surg 2011;141:318-21

$0022-5223 / \$ 36.00$

Copyright (c) 2011 by The American Association for Thoracic Surgery doi: $10.1016 /$ j.jtcvs.2010.10.013
}

rectangular system succeeded in providing a broad-based surgical education to interested and committed residents and certainly met the needs of society after World War II when the economy broadened, the population grew, medical knowledge exploded, technology improved, the demand for better health care expanded, and surgical specialization evolved. As a specialty, cardiothoracic surgery was in its infancy after World War II. We were in an age of clinical experimentation and reporting, we created surgical societies for continuing medical education, and there was little governmental or societal regulation. The creative environment after the war was intense, and no surgical specialty grew more dramatically than cardiothoracic surgery.

The amalgam of bright minds, daring personalities, and new technologies such as cardiopulmonary bypass (1953) expanded the specialty beyond lung and esophageal disease and fueled a new cardiac surgical profession. New operations for congenital heart disease, valvular heart disease, and atherosclerotic heart and aortic disease and transplantation were conceived and implemented in a very short historical time period. Thoracic surgery also expanded, although somewhat later, with the introduction of endoscopic surgery, lung volume reduction surgery, and lung transplantation. With the signing of the Social Security Act of 1965 , Medicare and Medicaid were established to pay for operations such as coronary artery bypass grafting, pulmonary resection for cancer, and transplantation. The combination of a bright and expanding future, little competition, high societal esteem, and excellent remuneration attracted the best and the brightest of surgical minds and hands to cardiothoracic surgery. Well-recognized surgical leaders then came out of leading cardiothoracic surgery institutions, such as Johns Hopkins, Duke University, Stanford University, and Cleveland Clinic. Cardiothoracic surgery was flourishing.

Jump ahead a short 60 years to 2010. The future for cardiothoracic surgery is less certain with all sorts of real or perceived threats. We no longer seem to be attracting the best and brightest to our profession, residencies are going unmatched, more foreign medical graduates are applying for US residencies, and more graduating residents are failing both the "qualifying" and the "certifying" ABTS examinations. Intravascular technologies continue to evolve with the introduction of intracoronary artery stenting, thoracic endovascular stenting for larger vessel aortic disease, and now the potential for intravascular approaches to aortic and mitral valvular heart disease. Competing technologies and multiple alternative training algorithms are competing in our classic domains of care, and the surgical future of 
cardiac surgery at least is perceived as uncertain. The biology of atherosclerosis has been influenced by new medical therapies, better control of diabetes and hypertension, smoking cessation programs, and educational access via the Internet. The cardiac surgery staple of coronary artery bypass grafting has shown a significant decline in volume during the last 8 years. The current environments to practice cardiothoracic surgery have also changed fairly dramatically. All experimentation, basic science or clinical, and even chart reviews must undergo the scrutiny of internal review boards, and the introduction of new medical technology or drugs must pass stringent Food and Drug Administration review. Since the publication of "To Err Is Human: Building a Safer Health System" by the Institute of Medicine in $2000,{ }^{3}$ society has come to realize that medical errors contribute to complications and death. This has placed a set of regulations and priorities on the health care system with new emphasis on compliance, patient safety, medical outcomes, and practice guidelines. With the explosion of the communication impact of the Internet and proliferation of administrative and professional databases, every procedure we perform and every outcome we achieve as surgeons is catalogued, reviewed, or analyzed. We now live in a world of regulation much different than the earlier environment when surgery grew so rapidly.

Other factors of change have also occurred. Some $50 \%$ of medical school graduating classes are women, yet in cardiothoracic surgery less than $5 \%$ of our trainees and professional physician workforce are women. The average length of training to become a cardiothoracic surgeon is 7 to 9 years after medical school, and the average debt of a graduating surgical resident is more than $\$ 100,000$. The millennium generation college graduate has different work ethic and lifestyle priorities compared with the overachieving, goal-oriented, post-war baby boomers. Information is available almost instantaneously. The theories of adult learning have evolved, and learning is accomplished much differently than in the past. Whereas for so many years information was obtained by going to the library to read books or journal articles, or knowledge and experience was imparted at the bedside from a dedicated professor, now the emphasis is more on asynchronous learning from databases or electronically online. Information is quickly available on the Internet, but knowledge is more difficult to set into the cortices of our brains. Opportunistic learning and "just in time" preparation are preferred by the modern trainee to more analytic learning. The volume of routine "bread and butter" surgery has decreased, and at a time when the complexity of each case has increased, so the teaching environment in the operating room has become more stressed. Faculty are distracted with the need to generate revenue for the hospital and department, efficiency in the operating room is valued, outcomes are more closely analyzed, and education has been threatened to become marginalized in priority.
Cardiothoracic surgery has classically been founded on relatively invasive "open" surgical approaches to intrathoracic disease. During the last decade, however, minimally invasive alternatives have evolved in both thoracic and cardiac disease. Therapeutic bronchoscopy, endoscopic lung resection, pleural drainage, and even robotic approaches to esophageal or lung resection have emerged. Off-pump coronary artery bypass, endovascular approaches to thoracic aortic disease, hybrid approaches to congenital heart disease, and now endovascular approaches to valvular heart disease have evolved. The vast number of earlier or classically trained surgeons, have not been exposed or trained in these new surgical options. Catheter-based skills have been more formally trained in invasive cardiology or radiology training programs, so as new technology has been introduced most of our current workforce do not have the new skills to easily learn, practice, or become credentialed in these new technical skills. Current surgeons in practice are therefore threatened by new competition and diminishing volumes from other specialties. Postgraduate surgical education has historically been under the responsibility of our societies and to a lesser degree industry with maintenance of certification under the aegis of the ABTS and credentialing more local through hospital structures. Societies such as the STS and AATS have put on numerous postgraduate programs that are observational and expose the specialty to new technology and new skills. Societies have not been in the business of the training of new skills. Industry must introduce their new technology and establish some competency training standards, but often this is initially done under a highly regulated clinical trial with limited access.

Maintenance of certification is a complex topic undergoing national scrutiny, but traditionally, boards such as the ABTS have made it relatively simple for practicing physicians to maintain their certification once in practice. Local credentialing and even national credentialing guidelines can be more complex by creating unrealistic standards with the potential for individual or specialty "turf wars." Industry is also increasingly scrutinized on the basis of issues of conflicts of interest if they move beyond training for individual devices or pharmaceutical drugs. How then do we introduce new skills, new technology, and new devices to the practicing cardiothoracic surgeon?

\section{SOLUTIONS}

There are no simple solutions, but progress is being made. In 2008, the leadership of 4 of the important societal organizations in cardiothoracic surgery, the ABTS, STS, AATS, and Thoracic Surgery Foundation of Research and Education, made an investment in education to deal with some of these issues by creating the Joint Council for Thoracic Surgery Education (JCTSE). Dr Edward Verrier was selected to be the Surgical Director of Education for the 
JCTSE, and his team has worked for the last year and a half to identify critical areas in surgical education that needed improvement or alternative approaches. The thrust of activity to date has focused on resident and postgraduate education.

\section{RESIDENT TRAINING}

For resident education, a number of initiatives have occurred as the result of collaboration among the JCTSE, the Thoracic Surgery Directors Association (TSDA), the ABTS, and the societies. New curricular options in resident education have evolved. The ABTS dropped the prerequisite that all entering cardiothoracic residents had to be certified by the American Board of Surgery. This meant that all incoming residents had to complete a 5-year General Surgery residency and pass their boards in General Surgery before being eligible to take the ABTS examinations. Most residencies were therefore either $5 / 2$ or $5 / 3$ in duration with cardiothoracic training either 2 or 3 years depending on local case volumes and program accreditation by the Thoracic Surgery Residency Review Committee. A 4/3 option was created within a single institution allowing a resident to still sit for both Boards but complete his/her combined training in 7 years with a greater emphasis on thoracic surgery in the last 4 years. A 6-year integrated option now exists by matching students right out of medical school and finishing training in 6 years without eligibility for American Board of Surgery certification. Currently, 9 cardiothoracic training programs have adopted this option, with the first graduates from Stanford expected to graduate in 2014. This option has become popular with medical students who have similar options in vascular and plastic surgery, with the 2010 match seeing approximately 100 applicants for these 9 positions. We hope this will once again allow us to attract the "best and the brightest" to cardiothoracic surgery. A complete integrated curriculum syllabus is being created for these residents with a greater emphasis on new skills, such as catheter-based and endovascular training and greater use of simulation. We believe this may be the preferred training option for the future. The current 2-year curriculum created by the TSDA in 2005 is being updated with improved PowerPoint presentations, new learning options (eg, blended classrooms online), and user, content, and learning management systems. The TSDA has held an educational "boot camp," supported in large part by the JCTSE, for incoming residents over the last 3 years whereby new residents are exposed to various simulation stations in a 2.5-day period in July taught by senior faculty in a nonthreatening, nurturing environment (bronchoscopy, mediastinoscopy, lobectomy, cannulation techniques, coronary artery anastomoses, and cardiopulmonary bypass). We would like to see this program expanded regionally so that all incoming residents in both the classic $5 / 2$ and $5 / 3$, and the integrated I- 6 residents will able to par- ticipate. The JCTSE held its first "Educate the Educators" symposium this past July, whereby 40 senior and junior faculty were taught adult learning theory, how to teach in the operating room, curriculum development and implementation, how to improve assessment skills, and how to use the science of education as a faculty advancement tool. We hope this will lead to the creation of an ongoing "Institute of Thoracic Surgery Education" whereby we elevate thoracic surgery education in priority, quality, and reward. Similar curricular efforts are being instituted for congenital heart surgery training programs and cardiothoracic critical care.

\section{POSTGRADUATE EDUCATION}

In postgraduate education, the JCTSE is looking to define new training options for the resident just coming out of training who is not exposed to new skills or the practicing surgeon wanting to expand his/her skills. We have arbitrarily split our organization into 3 areas: minimally invasive approaches to thoracic disease, minimally invasive approaches to cardiac disease, and endovascular approaches to aortic and valvular heart disease. Minimally invasive approaches to thoracic disease would include but not be limited to endobronchial ultrasound, therapeutic bronchoscopy, thoracoscopic lung resection, and robotics. Minimally invasive approaches to cardiac disease would include off-pump coronary revascularization, small-incision valvular heart disease, and robotics. Endovascular approaches would include thoracic endovascular aortic replacement and transaortic valve insertion. Credentialing standards have been written or are being written from a thoracic surgery perspective for each of these areas where indicated. We have catalogued new training options outside the classic residency training options. We are in the process of creating curriculum, defining program quality standards, and optimizing the educational environments for these new training options. We believe there will be a need for both 3-month minifellowships and 1-year full fellowships depending on prior trainee experience. The funding of such fellowships is being actively discussed so that a viable business plan will emerge and limited new fellowship options will be available by July 2011. In conjunction with the combined STS and AATS Task Force on New Technology, the JCTSE will cosponsor an introductory course in transaortic valve insertion in December 2010.

\section{SIMULATION/ELECTRONIC LEARNING}

Two overlapping areas of interest that both resident and postgraduate education have to deal with is the effective use of simulation and the electronic "thoracic surgery educational universe." Simulation is used extensively in the airline industry and business. In light of the new emphasis on improving surgical safety in an increasingly complex operative environment, the pressure to introduce both technical 
skill and situational simulation as learning tools is increasing. Simulation can be both low fidelity and high fidelity but often takes considerable time and expense to develop. Simulation has been slow to find a comfortable and useable place in thoracic surgery education. The expansion of the boot camp concept and additional pressure from both the ABTS and Thoracic Surgery Residency Review Committee to implement such programs provide the foundation on which we are building this effort. Almost every other surgical specialty has made significant financial and faculty investments in improving electronic learning. An entire billion dollar industry has evolved over the last decade just related to learning online education. User management, content management, and learning management systems are widely used in almost every learning environment from grade school to college and medical school, as well as industry. Examples include open source systems such as "Moodle" and propriety systems such as "Blackboard." Such educational sites online must be usable and easy to navigate, must conform to platform standards, and must show value over time. The creation and sustainability of such learning systems require foresight, investment, and commitment. The JCTSE is currently investigating such options for cardiothoracic surgery.

The specialty of cardiothoracic surgery has evolved, and education has evolved. Learners learn differently, and therefore teachers must teach differently. New technology and skill sets are necessary for thoracic surgery to grow and flourish. We must change even though change is not easy. The Joint Council on Thoracic Surgery Education, Inc, is an investment in our future.

\section{References}

1. Grillo HC. To impart this art: the development of graduate surgical education in the United States. Surgery. 1999;125:1-14.

2. Grillo HC, Edward D. Churchill and the "rectangular" surgical residency. Surgery. 2004; 136:947-52.

3. Kohn LT, Corrigan JM, Donaldson MS. To err is human: building a safer health system. In: Institute of Medicine. Washington, DC: National Academy Press; 2000 . 